\title{
GENERALIZED SWAN'S THEOREM AND ITS APPLICATION
}

\author{
P. MANOHARAN
}

(Communicated by Thomas Goodwillie)

\begin{abstract}
Swan's theorem verifies the equivalence between finitely generated projective modules over function algebras and smooth vector bundles. We define $A^{(r)}$-maps that correspond to usual non-linear differential operators of degree $r$ under the equivalence of Swan's theorem and thus generalize Swan's theorem to include non-linear differential operators as morphisms. An $A^{(r)}$ manifold structure is introduced on the space of sections of a fiber bundle through charts with $A^{(r)}$-maps as transition homeomorphisms. A characterization for all the smooth maps between the spaces of sections of vector bundles, whose $k$ th derivatives are linear differential operators of degree $r$ in each variable, is given in terms of $A^{(r)}$-maps.
\end{abstract}

\section{INTRODUCTION}

Let $A$ be the algebra of smooth real valued functions on a smooth compact connected finite dimensional manifold $K$. The space of smooth maps from $K$ to another finite dimensional manifold, or more generally, the space of sections of a fiber bundle over $K$, can be equipped with an $A$-manifold structure as described in [2] or [3]. Unfortunately an $A$-manifold structure is insufficient if one also wishes to involve the derivatives of mappings. For this purpose Kobayashi [2] introduced an $A^{(r)}$-manifold structure in terms of sheaves and studied certain differential geometric properties. An $A^{(r)}$-manifold structure is used on the space of sections because the usual Fréchet space structure is sometimes too general to be effective, mostly due to the failure of the inverse function theorem in the Fréchet category.

In this paper we describe the $A^{(r)}$-manifold structure more directly in terms of charts with finitely generated projective modules over $A$ as model spaces. The transition homeomorphisms, which we call $A^{(r)}$-maps, are introduced in the next section by further extending the non-linear version of Swan's theorem given in [3]. The results of [3] can be generalized to $A^{(r)}$-maps. For any fixed positive integers $k$ and $r$, we characterize all the maps between the spaces of sections of smooth vector bundles over $K$ whose $k$ th order derivatives are linear differential operators of degree $r$ in each variable.

Received by the editors October 12, 1993.

1991 Mathematics Subject Classification. Primary 58D15.

Key words and phrases. Non-linear differential operators, $A^{(r)}$-map, $A^{(r)}$-manifold.

The author was partially supported by a grant from KSU Research Council. 


\section{SWAN'S THEOREM}

Swan's theorem of [4] is generalized in [3] and is shown that there is an equivalence between the category of smooth vector bundles over $K$ with nonlinear (means not necessarily linear) fiber preserving maps as morphisms and the category of finitely generated projective $A$-modules with $A$-maps as morphisms. Let us recall the definition of $A$-maps from [3].

Definition 2.1. Let $\mathscr{E}_{1}, \mathscr{E}_{2}$ be any two finitely generated projective $A$-modules with Fréchet structures obtained from $A$. A smooth map $\Phi: \mathscr{U} \rightarrow \mathscr{E}_{2}$ defined on an open subset $\mathscr{U} \subseteq \mathscr{E}_{1}$ is called an $A$-map if the derivative $D_{s} \Phi: \mathscr{E}_{1} \rightarrow \mathscr{E}_{2}$ at each $s \in \mathscr{U}$ is $A$-linear.

Lemma 2.2. An R-linear map $\Psi: \mathscr{E}_{1} \rightarrow \mathscr{E}_{2}$ is A-linear iff $\Psi\left(\mathbf{m} \mathscr{E}_{1}\right) \subseteq \mathbf{m} \mathscr{E}_{2}$ for every maximal ideal $\mathbf{m}$ of $A$.

Proof. Every maximal ideal $\mathbf{m}$ of $A$ is given by $\mathbf{m}=\{f \in A \mid f(x)=0\}$ for some fixed $x \in K$. By the equivalence of Swan's theorem if we identify a finitely generated projective module $\mathscr{E}$ with the space of sections of a vector bundle, notice that for any $s \in \mathscr{E}, s \in \mathbf{m} \mathscr{E}$ if and only if $s(x)=0$.

Let us assume that $\Psi$ is an $R$-linear map such that $\Psi\left(\mathbf{m} \mathscr{E}_{1}\right) \subseteq \mathbf{m} \mathscr{E}_{2}$ for every maximal ideal $\mathbf{m}$ of $A$. For each $s \in \mathscr{E}_{1}, f \in A$ and $x \in K, f s-f(x) s \in \mathbf{m} \mathscr{E}_{1}$ where $\mathbf{m}$ is the maximal ideal associated to $x$. Therefore $\Psi(f s-f(x) s)=$ $\Psi(f s)-f(x) \Psi(s) \in \mathbf{m} \mathscr{E}_{2}$ which implies that $\Psi(f s)(x)=f(x) \Psi(s)(x)$ and thus $\Psi$ is $A$-linear. The converse part is obvious.

This leads us to the following definition.

Definition 2.3. An $R$-linear map $\Psi: \mathscr{E}_{1} \rightarrow \mathscr{E}_{2}$ is called a differential operator of degree $r$ if $\Psi\left(\mathbf{m}^{r+1} \mathscr{E}_{1}\right) \subseteq \mathbf{m} \mathscr{E}_{2}$ for every maximal ideal $\mathbf{m}$ of $A$. A map $\Phi: \mathscr{U} \rightarrow \mathscr{E}_{2}$ is called an $A^{(r)}-m a p$ if the derivative $D_{s} \Phi$ at every $s \in \mathscr{U}$ is a differential operator of degree $r$.

We show that $A^{(r)}$-maps correspond to a usual non-linear differential operator of degree $r$ under the correspondence of Swan's theorem between finitely generated projective $A$-modules and smooth vector bundles over $K$.

Let $E_{1}, E_{2} \rightarrow K$ be any two smooth vector bundles over $K$ and let $j^{r} E_{1}$ be the $r$-jet bundle of $E_{1}$. A non-linear differential operator of degree $r$ is a smooth map $\Phi: \Gamma E_{1} \rightarrow \Gamma E_{2}$ between the spaces of sections, induced by a smooth fiber preserving map $\phi: j^{r} E_{1} \rightarrow E_{2}$ and defined as $\Phi(s)(x)=\phi$ 。 $\left(j^{r} s\right)(x)$ for each $s \in \Gamma E_{1}, x \in K$.

Lemma 2.4. If $\mathbf{m}=\{f \in A \mid f(x)=0\}$, then $s \in \mathbf{m}^{r+1} \Gamma E_{1}$ if and only if $j^{r} s(x)=0$.

Proof. Locally at $x$, for every $h \in \Gamma E_{1}$ and $f_{i} \in \mathbf{m}$ we have

$$
D^{j}\left(f_{1} \cdots f_{r+1} h\right)(x)=\sum_{\alpha+\beta=j}\left(\begin{array}{l}
j \\
\alpha
\end{array}\right) D^{\alpha}\left(f_{1} \cdots f_{r+1}\right) \cdot D^{\beta}(h)(x)=0 \quad \forall j \leq r .
$$

Hence $s \in \mathbf{m}^{r+1} \Gamma E_{1} \Rightarrow j^{r} s(x)=0$. On the other hand let us suppose that $j^{r} s(x)=0$. Let $\left\{s_{1}, \ldots, s_{n}\right\}$ be a local base of sections at $x$. Then $s=\sum f_{i} s_{i}$ for some $f_{i} \in A . j^{r} s(x)=0$ implies that $D^{j} f_{i}(x)=0$ for all $j \leq r$. Therefore each $f_{i}$ belongs to $\mathbf{m}^{r+1}$ by Taylor formula and hence $s \in \mathbf{m}^{r+1} \Gamma E_{1}$. 
Lemma 2.5. Every non-linear differential operator of degree $r$ is an $A^{(r)}$-map.

Proof. Let $\Phi$ be a non-linear differential operator induced by $\phi: j^{r} E_{1} \rightarrow E_{2}$. $\phi$ yields the map $\Gamma \phi: \Gamma\left(j^{r} E_{1}\right) \rightarrow \Gamma E_{2}$ defined by $(\Gamma \phi)(\eta)=\varphi \circ \eta$ for each $\eta \epsilon$ $\Gamma\left(j^{r} E_{1}\right)$. For every $h \in \Gamma E_{1}$ one can verify that $D_{s} \Phi(h)(x)=D_{j^{r} s}(\Gamma \phi)\left(j^{r} h\right)(x)$. Moreover if $h \in \mathbf{m}^{r+1} \Gamma E_{1}$, then $j^{r} h(x)=0$ by the above lemma. Since $D_{j^{\prime} s}(\Gamma \phi)$ is $A$-linear by Lemma $2.2[3], j^{r} h(x)=0$ implies $D_{j r_{s}}(\Gamma \phi)\left(j^{r} h\right)(x)=$ 0 . Thus $\left(D_{s} \Phi\right)\left(\mathbf{m}^{r+1} \Gamma E_{1}\right) \subseteq \mathbf{m} \Gamma E_{2}$ for every maximal ideal $\mathbf{m}$ and hence $\Phi$ is an $A^{(r)}$-map.

Conversely,

Proposition 2.6. If $\Phi: \Gamma E_{1} \rightarrow \Gamma E_{2}$ is an $A^{(r)}$-map, then $\Phi$ is a non-linear differential operator of degree $r$.

Proof. First notice that for every $s, t \in \Gamma E_{1}$ and $x \in K, \Phi(s)(x)=\Phi(t)(x)$ whenever $j^{r} s(x)=j^{r} t(x)$. The proof of this fact is similar to that of Lemma 2.4 [3]. For, let $\left(E_{2}\right)_{x}$ be the fiber of $E_{2}$ over $x$. Define $\eta: R \rightarrow\left(E_{2}\right)_{x}$ by $\eta(\omega)=\Phi[s+\omega(t-s)](x)$. Then $d \eta / d \omega=\left(D_{s+\omega(t-s)} \Phi\right)(t-s)(x)=0$ because $\Phi$ is an $A^{(r)}$-map and $t-s \in \mathbf{m}^{r+1} \Gamma E_{1}$ by Lemma 2.4. Therefore $\Phi(s)(x)=\eta^{(0)}=\eta(1)=\Phi(t)(x)$.

The required map $\phi: j^{r} E_{1} \rightarrow E_{2}$ can be defined as follows: for any element $e \in j^{r} E_{1}$, choose $s \in \Gamma E_{1}$ such that $e=j^{r} s(x)$ and define $\phi(e)=\Phi(s)(x)$ which is independent of the choice of $s$ by the fact just noticed above and hence $\phi$ is well-defined. Obviously $\Phi(s)(x)=\phi \circ j^{r} s(x)$ for each $s \in \Gamma E_{1}$ and $x \in K$.

Thus we have further extended Swan's theorem:

Theorem 2.7. There is an equivalence between the category of smooth vector bundles with non-linear differential operators of degree $r$ as morphisms and the category of finitely generated projective A-modules with $A^{(r)}$-maps as morphisms.

We now describe an $A^{(r)}$-manifold structure on the space of sections.

Definition 2.8. An $A^{(r)}$-manifold is a Hausdorff topological space which is locally modeled on finitely generated projective $A$-modules such that all the transition maps are $A^{(r)}$-maps. Since every $A^{(r)}$-map is also an $A^{(r+1)}$-map, every $A^{(r)}$-manifold is an $A^{(r+1)}$-manifold and similarly every $A$-manifold is an $A^{(r)}$ manifold for each $r$. As an example, if $M \stackrel{\pi}{\rightarrow} K$ is a surjective submersion, the space of section $\Gamma M=\{f: K \rightarrow M \mid \pi \circ f=$ id $\}$ is an $A$-manifold by Proposition 3.5 [3] and hence an $A^{(r)}$-manifold for each $r$.

Since all $A^{(r)}$-maps and $A^{(r)}$-manifolds are tame, the Nash-Moser inverse function theorem holds for $A^{(r)}$-maps [1]. But in the case of $A$-manifolds and $A$-maps one can directly verify the usual inverse function theorem.

Theorem 2.9. If $\Phi: \Gamma E \rightarrow \Gamma E$ is an A-map such that $D_{s} \Phi$ is an isomorphism at some $s \in \Gamma E$, then $\Phi$ is a local diffeomorphism at $s$.

Proof. Since $\Phi$ is an $A$-map, there exists a unique fiber preserving map $\phi: E \rightarrow$ $E$ such that $\Phi(h)=\phi \circ h$ for each $h \in \Gamma E$. One can verify by direct computation that $D_{S} \Phi$ is an isomorphism implies that $D_{s(x)} \phi$ is an isomorphism for each $x \in K$. By the finite dimensional inverse function theorem, $\phi$ is a 
local diffeomorphism at each $s(x)$. Hence $\phi$ is a fiber preserving local diffeomorphism on a tubular neighborhood of $s(K)$ which implies that $\Phi$ is a local diffeomorphism at $s$.

\section{Higher DERIVATIVES}

In this section we give a characterization of all smooth maps between the spaces of sections of smooth vector bundles over $K$ whose $k$ th derivative (for any fixed positive integer $k$ ) is a linear differential operator in each variable. This result generalizes Theorem 5.2 [3].

Theorem 3.1. Let $\Psi: \Gamma E_{1} \rightarrow \Gamma E_{2}$ be a smooth map. The $k$ th derivative

$$
D_{s}^{k} \Psi: \overbrace{\Gamma E_{1} \times \cdots \times \Gamma E_{1}}^{k} \rightarrow \Gamma E_{2}
$$

at each $s \in \Gamma E_{1}$ is a linear differential operator of degree $r$ in each variable if and only if $\Psi-T^{k-1}(\Psi)$ is an $A^{(r)}-$ map, where $T^{k-1}(\Psi)$ is the $(k-1)$ th degree Taylor polynomial of $\Psi$ at the zero section $s_{0}$.

Proof. Let $\Psi-T^{k-1}(\Psi)=\Phi$ be an $A^{(r)}$-map where

$$
\begin{aligned}
T^{k-1}(\Psi)(h)= & \Psi\left(s_{0}\right)+\left(D_{s_{0}} \Psi\right)(h)+\frac{1}{2 !}\left(D_{s_{0}}^{2} \Psi\right)(h, h) \\
& +\cdots+\frac{1}{(k-1) !}\left(D_{s_{0}}^{k-1} \Psi\right)(h, \ldots, h) .
\end{aligned}
$$

Since $D_{s}^{k}\left(T^{k-1} \Psi\right)=0$ at each $s$, we have $D_{s}^{k} \Psi=D_{s}^{k} \Phi . \Phi$ is an $A^{(r)}$-map implies that there exists a smooth map $\phi: j^{r} E_{1} \rightarrow E_{2}$ such that $\Phi(s)(x)=\phi$ 。 $j^{r} s(x)$ by Proposition 2.6. Let $h_{1}, \ldots, h_{k} \in \Gamma E_{1}$ and $h_{i} \in \mathbf{m}^{r+1} \Gamma E_{1}$ for some $1 \leq i \leq k$. Then $j^{r} h_{i}(x)=0$ by Lemma 2.4. Now $\left(D_{s}^{k} \Phi\right)\left(h_{1}, \ldots, h_{k}\right)(x)=$ $\left(D_{j^{\prime} s}^{k} \Gamma \phi\right)\left(j^{r} h_{1}, \ldots, j^{r} h_{k}\right)(x)=0$ and hence $\left(D_{s}^{k} \Psi\right)=\left(D_{s}^{k} \Phi\right)$ is a differential operator of degree $r$ in each variable.

Conversely, let $\Psi: \Gamma E_{1} \rightarrow \Gamma E_{2}$ be a smooth map such that $D_{s}^{k} \Psi$ is a differential operator of degree $r$ in each variable. We need to show that $\Psi-T^{k-1}(\Psi)$ is an $A^{(r)}$-map.

For $k=1$ the proof follows from the definition of $A^{(r)}$-maps. For $k \geq 2$ we use the induction hypothesis. Let $T^{k-1}(\Psi)=\sum_{p=0}^{k-1} \mu_{p}$ where $\mu_{p}(h)=$ $\frac{1}{p !}\left(D_{s_{0}}^{p} \Psi\right)(h, \ldots, h)$. Consider $\eta=\Psi-\mu_{k-1}$.

Claim. $D_{s}^{k-1} \eta$ is a differential operator of degree $r$ in each variable.

Since $D_{s}^{k-1} \eta$ is symmetric, it is enough to prove for the first variable. Let $s_{1}, \ldots, s_{k-1} \in \Gamma E_{1}$ and $j^{r} s_{1}(x)=0$. First notice either by direct computation or by Sublemma 5.4 [3] that $D_{s}^{k-1} \mu_{k-1}\left(s_{1}, \ldots, s_{k-1}\right)=D_{s_{0}}^{k-1} \Psi\left(s_{1}, \ldots, s_{k-1}\right)$. Then at $x$,

$$
\begin{aligned}
D_{s}^{k-1} \eta\left(s_{1}, \ldots, s_{k-1}\right) & =\left[D_{s}^{k-1} \Psi-D_{s}^{k-1} \mu_{k-1}\right]\left(s_{1}, \ldots, s_{k-1}\right) \\
& =\left[D_{s}^{k-1} \Psi-D_{s_{0}}^{k-1} \Psi\right]\left(s_{1}, \ldots, s_{k-1}\right) .
\end{aligned}
$$

The last expression is independent of $s$, because by differentiating $G(s)=$ $\left[D_{s}^{k-1} \Psi-D_{s_{0}}^{k-1} \Psi\right]\left(s_{1}, \ldots, s_{k-1}\right)$ with respect to $s$ in the direction of $\beta$, we get $\left(D_{s} G\right)(\beta)=\left(D_{s}^{k} \Psi\right)\left(s_{1}, \ldots, s_{k-1}, \beta\right)$ which is equal to 0 at $x$, since $j^{r} s_{1}(x)=0$ and $D_{s}^{k \Psi}$ is a differential operator of degree $r$ in each variable. 
Thus $j^{r} s_{1}(x)=0$ implies that $\left(D_{s}^{k-1} \eta\right)\left(s_{1}, \ldots, s_{k-1}\right)(x)=0$ and hence the claim.

By induction $\Psi-\mu_{k-1}=\eta=\Phi+T^{k-2}(\eta)$ where $\Phi$ is an $A^{(r)}$-map and $T^{k-2}(\eta)$ is the $(k-2)$-degree Taylor polynomial of $\eta$ at the zero section $s_{0}$ :

$$
\begin{aligned}
T^{k-2}(\eta) & =\sum_{p=0}^{k-2} \frac{1}{p !}\left(D_{s_{0}}^{p} \eta\right) \\
& =\sum_{p=0}^{k-2} \frac{1}{p !}\left(D_{s_{0}}^{p} \Psi\right)-\sum_{p=0}^{k-2} \frac{1}{p !}\left(D_{s_{0}}^{p} \mu_{k-1}\right) \\
& =T^{k-2}(\Psi)-0
\end{aligned}
$$

since $\left(D_{s_{0}}^{p} \mu_{k-1}\right)\left(h_{1}, \ldots, h_{p}\right)=\frac{1}{(k-p-1) !}\left(D_{s_{0}}^{k-1} \Psi\right)\left(h_{1}, \ldots, h_{p}, s_{0}, \ldots, s_{0}\right)=0$ for all $p \leq k-2$ by the multilinearity of $D_{s_{0}}^{k-1} \Psi$. Therefore $\Psi-\mu_{k-1}=$ $\Phi+T^{k-2}(\Psi)$ and hence $\Psi=\Phi+T^{k-2}(\Psi)+\mu_{k-1}=\Phi+T^{k-1}(\Psi)$ as needed.

Particularly for $k=2$, we get

Corollary 3.2. For any smooth map $\Psi: \Gamma E_{1} \rightarrow \Gamma E_{2}, D_{s}^{2} \Psi$ is a bilinear differential operator of degree $r$ for each $s \in \Gamma E_{1}$ (or in other words it is a linear differential operator separately on both variables) if and only if $\Psi=\Phi+L$ where $\Phi$ is an $A^{(r)}$-map and $L$ is a linear operator with respect to $R$.

\section{REFERENCES}

1. R. S. Hamilton, The inverse function theorem of Nash and Moser, Bull. Amer. Math. Soc. (N.S.) 7 (1982), 65-222.

2. S. Kobayashi, Manifolds over function algebras and mapping spaces, Tôkoku Math. J. (2) 41 (1989), 263-282.

3. P. Manoharan, A non-linear version of Swan's theorem, Math. Z. 209 (1992), 467-479.

4. R. G. Swan, Vector bundles and projective modules, Trans. Amer. Math. Soc. 105 (1962), 264-277.

Department of Mathematics, Kent State University, East Liverpool, Ohio 43920

E-mail address: manohara@msc.kent.edu 